\title{
LETTERS
}

\section{Conflicting evidence on vertical transmission and maternal SARS-CoV-2 infection}

The report from Kirtsman and colleagues, ${ }^{1}$ as well as some of the other reports they cite, add to growing support for vertical transmission of severe acute respiratory syndrome coronavirus 2 (SARS-CoV-2) within the maternal-fetal unit. Further corroborative publications have emerged as well, variably including evidence of viral RNA in placental membranes immediately at birth; histological chorioamnionitis; positive staining for the virus by immunohistochemical analyses of the fetal side of the placenta; presence of the virus on the fetal side of the placenta by in situ hybridization; detection of CD68+ histiocytes in areas of placental inflammation; and the presence of viral RNA in amniotic fluid pre-rupture and in neonatal blood..$^{2-6}$ Some have proposed that there are no characteristic findings in the placenta from mothers with active coronavirus disease 2019 (COVID-19), and some have not found unique differences in placental pathology after SARS-CoV-2 infection versus historical controls. ${ }^{7,8}$ However, other studies have reported both local placental inflammation and evidence of placental malperfusion and thrombosis, which coincide with general maternal dysfunction. ${ }^{3,9,10}$ Most reports of possible vertical transmission appear to occur near the time of delivery, but cumulative data are lacking. There are no convincing reports that propose a typical pattern and outcome of congenital infection arising from earlier trimester infections, and it is possible that compromise of placental integrity during maternal infection may be a risk factor for facilitating timely vertical transmission.

Several important factors may contribute to risk of infection in the peripartum period. Maternal disease can be severe and is compounded by the added risk of common comorbidities. Regardless of whether vertical transmission of SARSCoV-2 occurs, the newborn is at risk of acquiring infection in the early neonatal period. Both mother and newborn can act as vectors for infection in the community and in health care settings. Although respiratory foci for infection dominate, the presence of infectious virus in stool (enteric replication), in the vagina (fecal contamination), and in breast milk (more likely via contamination from other sources) has the potential to complicate the epidemiology of virus spread.

Although more data are required to fully understand how SARS-CoV-2 is spread from mother to child, prevention and control of infection among pregnant mothers and their offspring is imperative in the interim. This is especially important because both mothers and newborns may be relatively asymptomatic. The continuing search for definitive mechanisms of vertical transmission deserves our attention.

\section{Nevio Cimolai MD}

Physician, Faculty of Medicine, University of British Columbia and Children's and Women's Health Centre of British Columbia, Vancouver, BC
Cite as: CMAJ 2020 November 23;192: E1547. doi: 10.1503/cmaj.76892

\section{References}

1. Kirtsman M, Diambomba Y, Poutanen SM, et al. Probable congenital SARS-CoV-2 infection in a neonate born to a woman with active SARSCoV-2 infection. CMAJ 2020;192:E647-50.

2. Patanè L, Morotti $D$, Giunta MR, et al. Vertical transmission of COVID-19: SARS-CoV-2 RNA on the fetal side of the placenta in pregnancies with COVID-19 positive mothers and neonates at birth. Am J Obstet Gynecol MFM 2020;2:100145.

3. Facchetti F, Bugatti M, Drera E, et al. SARS-CoV-2 vertical transmission with adverse effects on the newborn revealed through integrated immunohistochemical, electron microscopy and molecular analyses of placenta. EBioMedicine 2020;59: 102951

4. Sisman J, Jaleel MA, Moreno W, et al. Intrauterine transmission of SARS-CoV-2 infection in a preterm infant. Pediatr Infect Dis J 2020;39:e265-7.

5. Penfield CA, Brubaker SG, Limaye MA, et al. Detection of severe acute respiratory syndrome coronavirus 2 in placental and fetal membrane samples. Am J Obstet Gynecol MFM 2020;2:100133.

6. Vivanti A, Vauloup-Fellous $C$, Prevot $S$, et al. Transplacental transmission of SARS-CoV-2 infection. Nat Commun 2020;11:3572.

7. Hecht JL, Quade B, Deshpande V, et al. SARS CoV-2 can infect the placenta and is not associated with specific placental histopathology: a series of 19 placentas from COVID-19-positive mothers. Mod Pathol 2020;33:2091-103.

8. Gulersen M, Prasannan L, Tam Tam H, et al. Histopathological evaluation of placentas after diagnosis of maternal SARS-CoV-2 infection. Am J Obstet Gynecol MFM 2020; 2:100211.

9. Shanes ED, Mithal LB, Otero S, et al. Placental pathology in COVID-19. Am J Clin Pathol 2020; 154:23-32.

10. Prabhu M, Cagino K, Matthews KC, et al. Pregnancy and postpartum outcomes in a universally tested population for SARS-CoV-2 in New York City: a prospective cohort study. BJOG 2020;127: 1548-56.

Competing interests: None declared. 